\title{
Desenvolvimento de um novo sensor para ácido ascórbico
}

\section{Development of a new ascorbic acid sensor}

\author{
Thiago da Cruz Canevari ${ }^{1}$; Rodrigo Ferrari de Castilho ${ }^{1}$; \\ Rení Ventura da Silva Alfaya²; Antonio Alberto da Silva Alfaya ${ }^{3}$
}

Resumo

O compósito $\mathrm{SiO}_{2} / \mathrm{SnO}_{2} /$ Fosfato, SSF, foi preparado pelo processo sol-gel. O corante redox azul de metileno foi imobilizado na superfície do SSF. Esse novo material foi utilizado na preparação de um eletrodo de pasta de carbono modificado (SSFAM) e suas características eletroquímicas foram estudadas pela técnica da voltametria cíclica. O sensor SSFAM apresentou um potencial formal de $-90 \mathrm{mV}$ vs ECS, uma excelente estabilidade quanto ao número de ciclos e quanto ao $\mathrm{pH}$ da solução de eletrólito suporte na faixa de 2,0 a 8,0. O sensor SSFAM foi utilizado para a determinação de ácido ascórbico (Vitamina C), com a utilização da técnica da cronoamperometria, e apresentou um tempo de resposta de 1,5 s, uma faixa linear de resposta entre 2,0 x $10^{-4} \mathrm{e} \mathrm{3,0}$ x $10^{-3} \mathrm{~mol} \mathrm{~L}^{-1}$, com um limite de detecção de $7,8 \times 10^{-6} \mathrm{~mol} \mathrm{~L}^{-1}$, considerando uma relação sinal/ ruído $=3$. Os resultados obtidos para amostras reais pelo sensor SSFAM foram comparados com os do método padrão de determinação de ácido ascórbico pelo reagente 2,6diclorofenilindofenol. A comparação dos resultados obtidos pelos dois métodos demonstrou que o sensor SSFAM possui grande potencial de utilização.

Palavras-chave: Sensor. Sol-gel. Ácido ascórbico.

\begin{abstract}
The $\mathrm{SiO}_{2} / \mathrm{SnO}_{2} / \mathrm{Phosphate}$ composite, SSF, was prepared by the sol-gel process. The redox dye methylene blue was immobilized on the SSF surface. This new material was utilized in the preparation of a modified carbon paste electrode (SSFAM) and its electrochemical characteristics were studied by the cyclic voltammetry technique. The SSFAM sensor presented a formal potential of $-90 \mathrm{mV}$ vs SCE, excellent stability regarding the number of cycles and regarding the $\mathrm{pH}$ of electrolyte support solution ranging from 2.0 to 8.0. The SSFAM sensor was utilized for the determination of ascorbic acid (Vitamin C), utilizing the chronoamperometry technique, and presented a response time of $1.5 \mathrm{~s}$, a linear response range between $2.0 \times 10^{-4}$ and $3.0 \times 10^{-3} \mathrm{~mol}$ $\mathrm{L}^{-1}$, with a detection limit of $7.8 \times 10^{-6} \mathrm{~mol} \mathrm{~L}^{-1}$, considering a relation signal $/$ noise $=3$. The results obtained for real samples by the SSFAM sensor were compared with those from the standard method for determination of ascorbic acid by the 2,6-dichlorophenilindophenol. The comparison of the obtained results by the two methods demonstrated that the SSFAM sensor has great utilization potential.
\end{abstract}

Key words: Sensor. Sol-gel. Ascorbic acid.

\footnotetext{
${ }^{1}$ Estudante do curso de Química, UEL - Londrina.

${ }^{2}$ Doutora - Departamento de Química, CCE - UEL. E-mail: reni@uel.br.

${ }^{3}$ Doutor - Departamento de Química, CCE - UEL. E-mail: alfaya@uel.br.
} 


\section{Introdução}

Atualmente, muitos procedimentos analíticos necessitam de instrumentações complexas e profissionais altamente especializados. Os sensores químicos são dispositivos pequenos, robustos, portáteis, de fácil manipulação e não necessitam da adição contínua de reagentes para a sua operação, e são, portanto, um elemento chave na instrumentação analítica para a determinação de importantes compostos químicos e biológicos em concentrações muito baixas (ALFAYA; KUBOTA, 2002). Baseados neste contexto, publicaram-se vários trabalhos (PALECEK, 1996; KUBOTA; GUSHIKEM; PEREZ, 1995; ROLISON, 1990), nos quais se utilizaram zeólitas, argilas, fosfato de zircônio e sílica modificada como suportes para imobilização de mediadores no desenvolvimento de sensores (PEREIRA; KUBOTA, 2004).

Os óxidos mistos do tipo $\mathrm{SiO}_{2} / \mathrm{M}_{\mathrm{x}} \mathrm{O}_{\mathrm{y}}$ foram utilizados com sucesso como substrato para fase orgânica ligada covalentemente em material de empacotamento de colunas para HPLC-RP resistentes a soluções alcalinas (SILVA; COLLINS, 1999; MELO; JARDIM, 1999; SILVA; COLLINS; COLLINS, 2000; MELO et al., 2000; SILVA; GUSHIKEM;COLLINS, 2001); e como adsorvente de espécies químicas (FUJIWARA; GUSHIKEM; ALFAYA, 2001) e imobilização de espécies eletroativas para a fabricação de sensores e biossensores eletroquímicos (PEREZ; NETO; KUBOTA, 2001; ROSATTO; KUBOTA; NETO, 1999). Todos esses substratos foram preparados pelo recobrimento de um filme fino do óxido metálico $\mathrm{M}_{\mathrm{x}} \mathrm{O}_{\mathrm{y}}$ sobre a superfície porosa da sílica gel pela técnica de enxerto (GUSHIKEM; ROSATTO, 2001).

Um dos principais problemas encontrados na sílica gel modificada com um filme fino de óxido metálico na superfície é a preparação, que exige precursores adequados para a reação de enxerto. Outro problema é o fato de as reações modificarem a superfície da sílica pré-existente, muitas vezes entupindo poros pequenos, de modo que, devido à quantidade de $\mathrm{Si}$ -
OH disponível para a reação de enxerto ser pequena, nunca resultam grandes quantidades de óxido metálico na superfície.

O processo sol-gel é um método de síntese que apresenta boa reprodutibilidade, alta homogeneidade, alta pureza, controle de volume e diâmetro de poros e por conseqüência um efetivo controle de área superficial específica. Ele pode ser realizado em aparelhagem simples e a baixa temperatura (ALFAYA; KUBOTA, 2002; WALCARIUS, 1998).

Recentemente, a síntese do óxido misto $\mathrm{SiO}_{2} / \mathrm{SnO}_{2}$ foi realizada pelo processo sol-gel (KURIHARA et al., 2004). Esse material é extremamente interessante, pois possui uma alta condutividade elétrica, devido à presença de nanopartículas de $\mathrm{SnO}_{2}$, que diminuem consideravelmente a resistência elétrica na matriz de sílica (CARDOSO et al., 2004).

O azul de metileno (Figura 1) é um corante orgânico da classe das fenotiazinas e está sendo extensivamente utilizado como mediador de elétrons, devido às suas propriedades eletrocatalíticas frente ao NADH (PESSOA et al., 1997) e ao ácido ascórbico (FERREIRA; GUSHIKEM; ALFAYA, 2000). O azul de metileno é um corante catiônico e é usado como espécie eletroativa, quando imobilizado sobre materiais fosfatados (PESSOA et al., 1997; ROCHA et al., 1997; MUNTEANU; KUBOTA; GORTON, 2001).

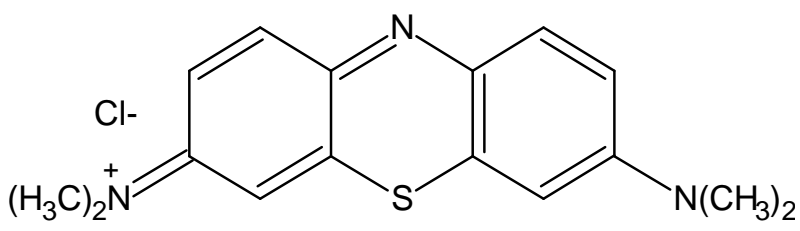

Figura 1. Estrutura do azul de metileno.

Este trabalho tem por objetivo estudar o comportamento eletroquímico do azul de metileno imobilizado sobre a superfície do compósito $\mathrm{SiO}_{2} /$ $\mathrm{SnO}_{2} /$ Fosfato pela técnica da voltametria cíclica, e, do mesmo modo, busca avaliar a potencialidade deste novo eletrodo como sensor para ácido ascórbico. 


\section{Parte Experimental}

Todos os reagentes utilizados neste trabalho foram de grau analítico. A água utilizada foi de qualidade Milli- $\mathrm{Q}_{\text {plus }}$ com resistividade de $18 \mathrm{~m} \Omega \mathrm{cm}^{-1}$.

\section{Preparação do xerogel $\mathrm{SiO}_{2} / \mathrm{SnO}_{2}, \mathrm{SS}$}

O óxido misto SS foi preparado conforme o procedimento descrito na literatura por Kurihara, et al. (2004).

\section{Preparação do compósito $\mathrm{SiO}_{2} / \mathrm{SnO}_{2} /$ Fosfato, $\mathrm{SSF}$}

Foram dispersas $500 \mathrm{mg}$ de $\mathrm{SS}$ em $50 \mathrm{~mL}$ de uma solução $0,5 \mathrm{~mol} \mathrm{~L}^{-1} \mathrm{de}_{3} \mathrm{PO}_{4}$. Após $12 \mathrm{~h}$ de repouso filtrou-se o compósito $\mathrm{SiO}_{2} / \mathrm{SnO}_{2} /$ Fosfato e lavou-se com água abundantemente e o material obtido foi colocado para secar na temperatura ambiente.

\section{Imobilização do azul de metileno sobre SSF}

Pesou-se 500 mg do compósito SSF, e dispersouse em $50 \mathrm{~mL}$ de solução de azul de metileno $1,0 \times 10^{-3} \mathrm{~mol} \mathrm{~L}^{-1}$ e deixou-se em repouso por $8 \mathrm{~h}$ na temperatura ambiente e no escuro. Em seguida, filtrou-se e lavou-se o compósito com água. O material foi deixado secar na temperatura ambiente e no escuro. O material assim obtido é o $\mathrm{SiO}_{2} / \mathrm{SnO}_{2} /$ Fosfato/Azul de Metileno, SSFAM.

\section{Análises químicas}

A quantidade de $\mathrm{Sn}(\mathrm{IV})$ no compósito SSF foi determinada pelo tratamento de $0,5 \mathrm{~g}$ de amostra com solução de HF $40 \%$ até a completa dissolução do material. Aproximadamente $150 \mathrm{~mL}$ de água foram adicionados e o metal foi precipitado com adição de solução de $\mathrm{NH}_{4} \mathrm{OH}$ concentrado. $\mathrm{O}$ resíduo obtido foi calcinado a $900^{\circ} \mathrm{C}$ por $12 \mathrm{~h}$ e pesado como $\mathrm{SnO}_{2}$.

$\mathrm{O}$ teor de fósforo foi obtido pelo tratamento de 0,5 g do compósito SSF com $10 \mathrm{~mL}$ de solução nítrico/ perclórico $(3: 1 \mathrm{v} / \mathrm{v})$ a quente, até quase a secura. $\mathrm{O}$ material foi lavado quantitativamente com água e filtrado. Todas as águas de lavagem foram recolhidas e colocadas em um balão volumétrico de $100 \mathrm{~mL}$. A determinação do fosfato deslocado foi realizada espectrofotometricamente pelo método do azul de molibdênio (STANDARD METHODS FOR THE EXAMINATION OF WATER AND WASTEWATER, 1992).

A quantidade de azul de metileno imobilizado sobre o compósito SSF foi determinado pela digestão de $0,100 \mathrm{~g}$ de SSFAM em $\mathrm{H}_{2} \mathrm{SO}_{4}$ concentrado e posterior determinação do teor de nitrogênio pelo método de Kejdhal.

\section{Medidas físicas}

Isotermas de adsorção foram determinadas em um equipamento ASAP 2010 na temperatura do nitrogênio líquido. Inicialmente, a amostra foi tratada a $100^{\circ} \mathrm{C}$ por $48 \mathrm{~h}$. A área superficial específica do material foi obtida com a utilização do método MP (WEBB; ORR, 1997) e o volume médio dos poros foi obtido utilizando-se a aproximação HorvathKavazoe (HORVATH; KAVAZOE, 1983).

\section{Medidas eletroquímicas}

O eletrodo de pasta de carbono foi preparado pela mistura de $15 \mathrm{mg}$ de grafite, $15 \mathrm{mg}$ do compósito SSFAM e $1 \mathrm{mg}$ de óleo mineral parafínico $\left(\mathrm{Nujol}^{\circledR}{ }^{\circledR}\right.$ ). A pasta foi então depositada na extremidade do eletrodo de trabalho como pode ser visto na Figura 2. 


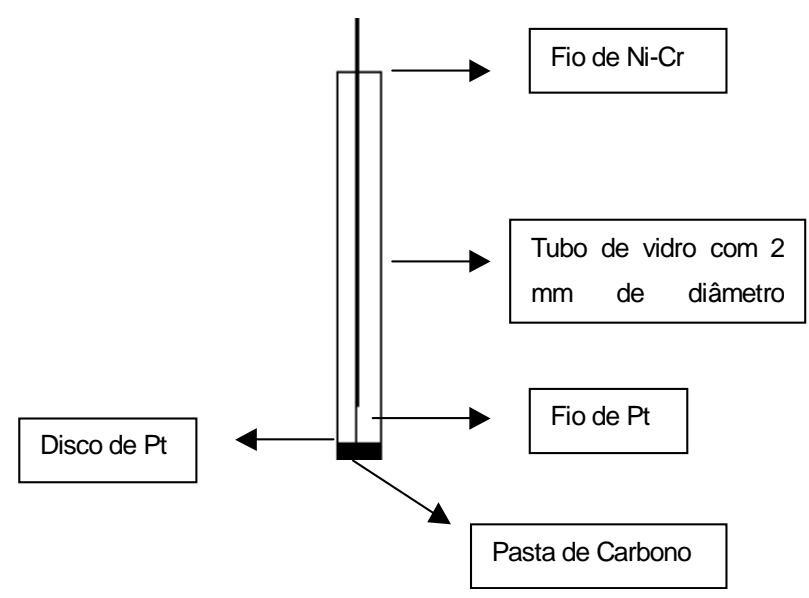

Figura 2. Esquema do eletrodo de pasta de carbono modificado.

As medidas eletroquímicas foram realizadas em um potenciostato MQPG-01, em conjunto com a interface MQ/12/8PG conectado a um microcomputador para controle do potencial e aquisição de dados. Todas as medidas foram efetuadas utilizando-se uma cela eletroquímica (com $5,0 \mathrm{~mL}$ de capacidade e termostatizada a $25^{\circ} \mathrm{C}$ ) de três eletrodos, onde um eletrodo de calomelano saturado (ECS) serviu como referência e uma espiral de platina como eletrodo auxiliar.

\section{Resultados e Discussão}

\section{Análises químicas e físicas}

O compósito $\mathrm{SS}$ apresentou as seguintes características. Um teor de 15,6\% (m/m) de Sn, uma área superficial específica $S_{B E T}=525 \mathrm{~m}^{2} \mathrm{~g}^{-1}$, um volume médio de poros de $0,28 \mathrm{~mL} \mathrm{~g}^{-1}$ e um diâmetro de poro de $1,5 \mathrm{~nm}$.

O teor de fósforo obtido no compósito SSF foi de $120 \mu \mathrm{mol} \mathrm{g}{ }^{-1}$ após determinação espectrofotométrica pelo método do azul de molibdênio (STANDARD METHODS FOR THE EXAMINATION OF WATER AND WASTEWATER, 1992). O teor de azul de metileno adsorvido sobre a superfície do compósito SSFAM foi de $56 \mu \mathrm{mol} \mathrm{g}{ }^{-1}$.

\section{Medidas eletroquímicas}

A Figura 3 mostra os voltamogramas cíclicos obtidos, com o uso de eletrodos modificados com SSFAM (A), SSF (B) e Grafite (C) em $\mathrm{KCl} 0,5$ mol $\mathrm{L}^{-1}$ com velocidade de varredura de $20 \mathrm{mV} \mathrm{s}^{-1}$. Observa-se claramente que o azul de metileno foi imobilizado sobre a superfície do compósito SSF. O potencial formal $\mathrm{E}^{0}$, definido como:

$\mathbf{E}^{0}=\left(\mathbf{E}_{\mathrm{pc}}+\mathbf{E}_{\mathrm{pa}}\right) / 2$

Equação 01

onde $\mathrm{E}_{\mathrm{pc}}$ é o potencial do pico catódico e $\mathrm{E}_{\mathrm{pa}}$ é o potencial de pico anódico. Para o azul de metileno imobilizado no SSF, o $\mathrm{E}^{0}$ ' foi de $-90 \mathrm{mV}$ em relação ao eletrodo de calomelano saturado (ECS), isso evidencia um forte deslocamento na direção positiva de potencial em relação ao potencial formal do azul de metileno em solução (-200 mV) (PESSOA et al., 1997). Esse deslocamento no potencial pode ser explicado pela presença dos grupos ácidos presentes na superfície da matriz que interagem com o azul de metileno estabilizando a forma reduzida.

A estabilidade do azul de metileno sobre a matriz SSF foi verificada medindo-se os valores das correntes anódicas e catódicas em função do número de ciclos realizados, na faixa de potencial de -500 a $400 \mathrm{mV}$ vs ECS, a uma velocidade de $20 \mathrm{mV} \mathrm{s}^{-1} \mathrm{em}$ $\mathrm{KCl}$ 0,5 mol L$^{-1}$ (Figura 4).

As intensidades das correntes de pico apresentam uma boa estabilidade, indicando que o azul de metileno não é lixiviado da superfície do compósito SSF ou decomposto durante o experimento.

Como a reação de oxirredução do azul de metileno envolve a participação de prótons, esta apresenta-se bastante sensível a mudanças de $\mathrm{pH}$ do meio (50 a $350 \mathrm{mV}$ na faixa de $\mathrm{pH} 2$ a 10) (PESSOA et al., 1997). Neste caso, a influência do pH do eletrólito suporte no valor do potencial formal, $\mathrm{E}^{0}$, , apresenta grande importância no estudo do comportamento do presente eletrodo modificado. 


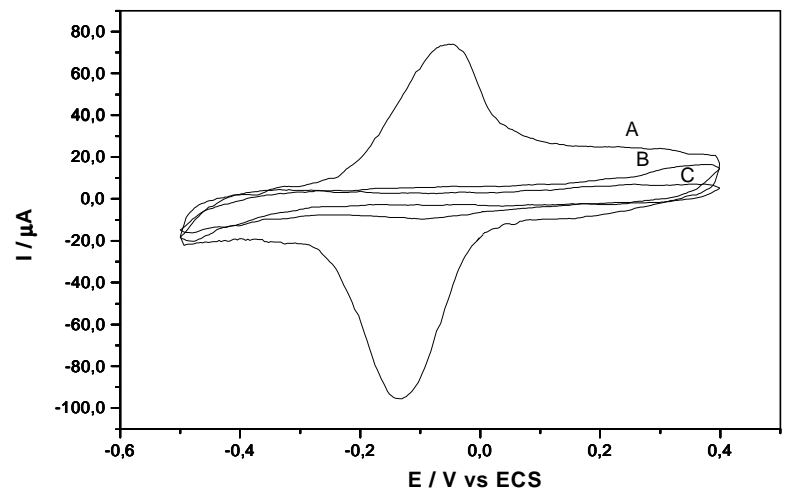

Figura 3. Voltamogramas cíclicos obtidos utilizando eletrodo de pasta de carbono modificado com SSFAM (A), SSF (B) e Grafite (C) em KCl 0,5 $\mathrm{mol} \mathrm{L}^{-1}$ e a $20 \mathrm{mV} \mathrm{s}^{-1}$.

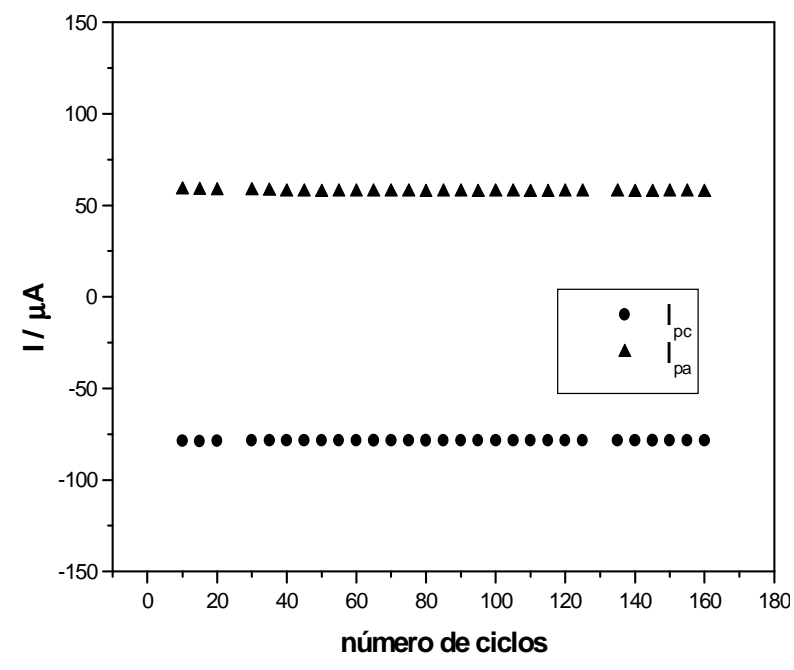

Figura 4. Estabilidade do eletrodo em $\mathrm{KCl} 0,5 \mathrm{~mol} \mathrm{~L}^{-1}$ a $20 \mathrm{mV} \mathrm{s}^{-1}$.

Um aspecto interessante observado durante o estudo do $\mathrm{pH}$ foi a não dependência do potencial formal com o pH do meio, na faixa de 3,0 a 8,0 (Figura 5), e nem mudanças significativas na forma dos voltamogramas cíclicos. Este comportamento difere completamente do observado para o azul de metileno em solução (PESSOA et al., 1997). Este fenômeno pode estar relacionado com a acidez superficial da matriz SSF produzida pelo processo sol-gel. Este mesmo resultado foi encontrado com o azul de metileno imobilizado em fosfato de zircônio (PESSOA et al., 1997).

Outra característica de grande importância na construção de sensores para amostras reais consiste na estabilidade das correntes de pico com o $\mathrm{pH}$ da solução. Nesse caso, foi também observado que os valores das correntes de pico, tanto anódica quanto catódica, mantiveram-se constantes em soluções de $\mathrm{KCl} 0,5 \mathrm{~mol} \mathrm{~L}^{-1}$ na faixa de $\mathrm{pH}$ entre 2,0 a 8,0 .

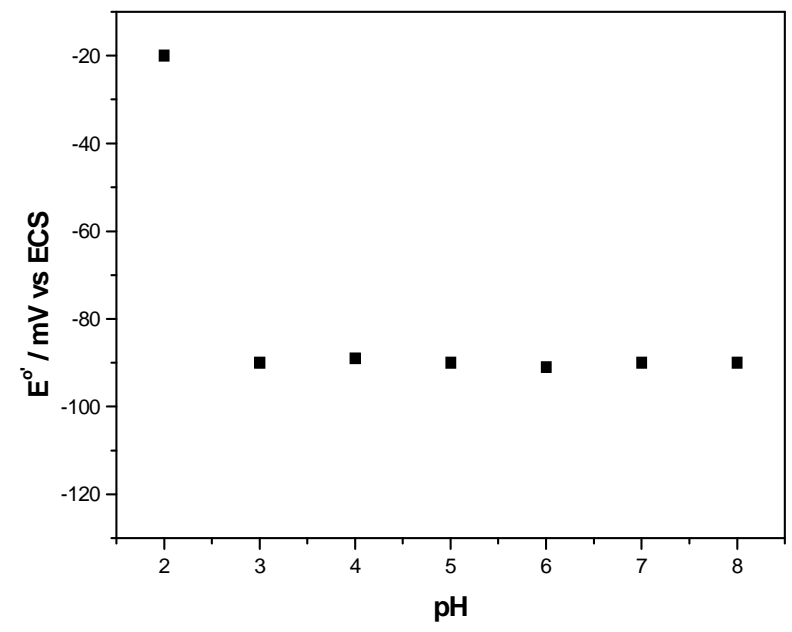

Figura 5. Dependência do potencial formal com o pH da solução do eletrólito suporte $\mathrm{KCl} 0,5 \mathrm{~mol} \mathrm{~L}^{-1}$, para o eletrodo de pasta de carbono modificado com SSFAM.

A reação redox do azul de metileno envolve a participação de prótons, com a diminuição do $\mathrm{pH}$, maior o número de prótons presentes no meio, de modo que o processo de transferência de elétrons se torna mais eficiente e, em consequiência, é maior a corrente observada. Entretanto, observa-se que nesse caso, tal fenômeno não ocorre, a corrente permanece constante na faixa de $\mathrm{pH}$ entre 2,0 a 8,0. Esse resultado reforça a hipótese de que quando imobilizado sobre a matriz SSF esta parece funcionar como uma proteção para a molécula de azul de metileno contra os efeitos do $\mathrm{pH}$ da solução do eletrólito suporte.

Com o intuito de verificar as propriedades eletrocatalíticas do presente eletrodo, realizou-se estudos utilizando o ácido ascórbico, $\mathrm{H}_{2} \mathrm{AA}$, como substrato. A oxidação eletroquímica do $\mathrm{H}_{2} \mathrm{AA}$ tem sido utilizada extensivamente como sistema modelo no estudo do efeito da modificação na sua atividade catalítica. Embora o ácido ascórbico apresente um 
potencial de oxidação relativamente baixo $(54 \mathrm{mV})$, a sua oxidação eletroquímica em eletrodos metálicos e de carbono ocorre em altos sobrepotenciais e pode, assim, sofrer a interferência de outras espécies oxidáveis, existentes em amostras biológicas (XING et al., 1992). A utilização dos chamados EQMs (eletrodos quimicamente modificados) tem possibilitado eletrooxidar o ácido ascórbico em potenciais consideravelmente mais baixos em relação aos eletrodos não modificados (DAUFARTAS; EVANS, 1980; ANDREOTTI; GUSHIKEM, 1992). A reação de oxidação de $\mathrm{H}_{2} \mathrm{AA}$, ocorre segundo um processo eletroquímico - químico (uma reação de transferência de elétrons seguida de um processo de hidratação), no qual o ácido ascórbico é oxidado irreversivelmente a ácido dehidroascórbico hidratado (HARTLEY, 1985).

Observa-se, pela Figura 6, que a oxidação do ácido ascórbico, com o uso do eletrodo de pasta de carbono modificado com SSFAM, ocorreu em $0,0 \mathrm{mV}$ vs ECS. A eletrooxidação do ácido ascórbico em eletrodo de grafite ocorre a $450 \mathrm{mV}$ vs ECS, e isso reforça o fato de que a eletrocatálise do ácido ascórbico se deve ao azul de metileno imobilizado e devido à natureza da matriz, ocorre uma redução de $450 \mathrm{mV}$ em relação a grafite, e, com isso, ocorre uma diminuição acentuada do sobrepotencial no eletrodo.

O tampão pH 7,0 utilizado na eletrooxidação do ácido ascórbico pelo eletrodo SSFAM foi o fosfato monobásico de potássio/KOH (MORITA; ASSUMPÇÃO, 1972).

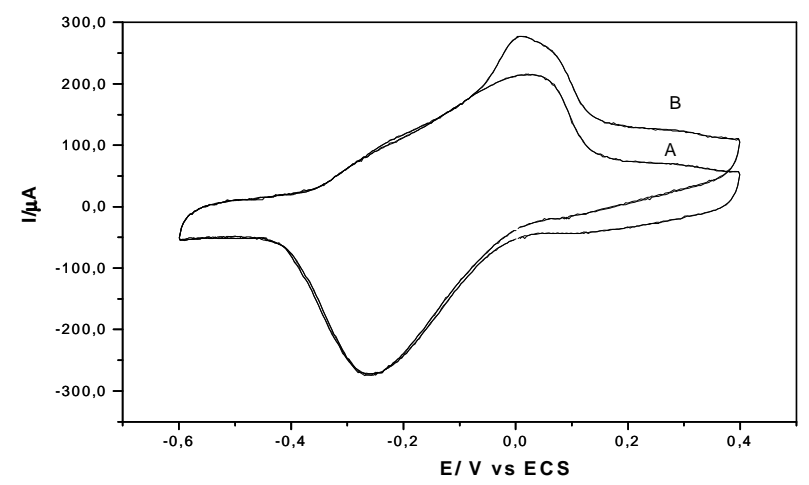

Figura 6. Voltamogramas cíclicos obtidos utilizando-se eletrodo de pasta de carbono modificado com SSFAM em KCl 0,5 $\mathrm{mol} \mathrm{L}^{-1}$ tamponado a pH 7,0 a uma velocidade de varredura de $20 \mathrm{mV} \mathrm{s}^{-1}$. (A) ausência de $\mathrm{H}_{2} \mathrm{AA}$ e (B) na presença de $\mathrm{H}_{2} \mathrm{AA}$.
A reação catalítica ocorrida na interface eletrodosolução pode ser descrita de maneira simplificada como abaixo:

$\mathrm{Na}$ interface eletrodo-solução:

$\mathrm{SSFAM}_{\mathrm{ox}}+\mathrm{H}_{2} \mathrm{AA} \rightarrow \mathrm{SSFAM}_{\mathrm{red}}+2 \mathrm{H}^{+}+\mathrm{DHAA}$

No eletrodo:

$\mathrm{SSFAM}_{\text {red }} \rightarrow \mathrm{SSFAM}_{\mathrm{ox}}+2 \mathrm{e}^{-}$

onde $\operatorname{SSFAM}_{\mathrm{ox}}$ e SSFAM ${ }_{\text {red }}$ correspondem às formas oxidada e reduzida do azul de metileno sobre a matriz SSF, respectivamente, $\mathrm{H}_{2} \mathrm{AA}$ o ácido ascórbico e DHAA a forma oxidada do ácido ascórbico (ácido dehidroascórbico).

\section{Obtenção da curva analítica para $\mathbf{H}_{2} \mathbf{A A}$ por cronoamperometria}

Fixando-se o potencial em $0,0 \mathrm{mV}$ vs ECS, foi obtida a curva analítica por amperometria para o $\mathrm{H}_{2} \mathrm{AA}$, por meio de adições sucessivas de alíquotas de $100 \mu \mathrm{L}$ de uma solução padrão de ácido ascórbico de concentração $\left[\mathrm{H}_{2} \mathrm{AA}\right]=1,0 \times 10^{-2} \mathrm{~mol} \mathrm{~L}^{-1} \mathrm{em} 5,0 \mathrm{~mL}$ de $\mathrm{KCl}$ 0,5 $\mathrm{mol} \mathrm{L}^{-1}$, tamponado a pH 7,0 (Figura 7).

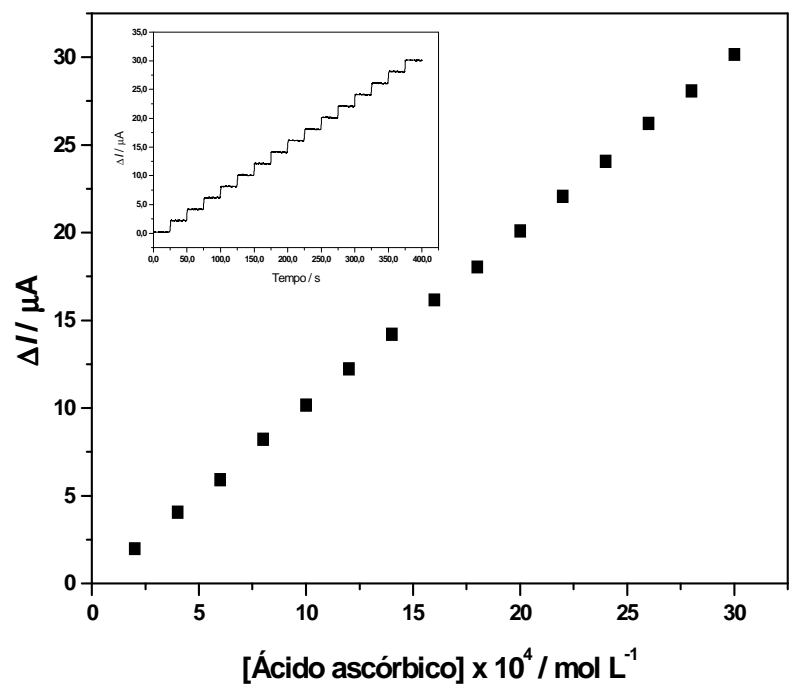

Figura 7. Dependência entre a corrente e a concentração de ácido ascórbico (curva analítica). Figura inserida: Cronoamperograma obtido após sucessivas adições de $100 \mu \mathrm{L}$ de ácido ascórbico 1,0x $10^{-2} \mathrm{~mol} \mathrm{~L}^{-1}$ em 5,0 mL de eletrólito suporte KCL 0,5 mol L' tamponado a $\mathrm{pH} 7,0$, fixando-se o potencial em $0,0 \mathrm{mV}$ vs ECS. 
A correlação linear obtida foi $\Delta I=0,078+$ $1,0024\left[\mathrm{H}_{2} \mathrm{AA}\right] \operatorname{com} \mathrm{r}=0,9999$ para $\mathrm{n}=15$. O limite de detecção foi de 7,8 x $10^{-6} \mathrm{~mol} \mathrm{~L}^{-1}$, considerando uma relação sinal/ruído $=3$. A reprodutibilidade com o mesmo eletrodo foi boa, apresentando um desvio padrão relativo de $1,8 \%$ para $n=15$. A média do tempo de resposta do eletrodo, medido após várias adições do substrato na cela eletroquímica, foi de cerca de 1,5 s. O eletrodo apresentou-se bastante estável, permitindo o seu uso por pelo menos 6 meses com utilização diária, sem significativas mudanças em sua resposta.

\section{Determinação do teor de ácido ascórbico em amostras reais}

Com o intuito de testar a potencialidade analítica do eletrodo de pasta de carbono SSFAM como um sensor eletroquímico para ácido ascórbico em amostras reais, a quantidade de vitamina $\mathrm{C}$ em tabletes, adquiridos em farmácias da região de Londrina, foi determinada pela utilização da técnica de cronoamperometria com potencial fixo em $0,0 \mathrm{mV}$ vs ECS.

Para a determinação do ácido ascórbico nos tabletes, as amostras foram preparadas como indicado pelo método padrão e os valores obtidos foram comparados com os obtidos pela utilização do reagente 2,6-diclorofenilindofenol (LINDQUIST, 1975). Os resultados estão sumarizados na Tabela 1.

Os resultados obtidos pelo sensor SSFAM apresentam boa concordância com os obtidos pelo método padrão, indicando que o mesmo é bastante promissor na construção de um sensor para ácido ascórbico.

Tabela 1 - Determinação de ácido ascórbico em tabletes usando o sensor SSFAM e comparando os resultados com os obtidos pelo método padrão.

\begin{tabular}{cccc}
\hline Amostras & Teor declarado / g & Método padrão $/ \mathrm{g}$ & SSFAM $^{\mathrm{b}} / \mathrm{g}$ \\
\hline A & 1,0 & $1,021 \pm 0,004$ & $1,020 \pm 0,004$ \\
B & 1,0 & $1,008 \pm 0,004$ & $1,001 \pm 0,005$ \\
C & 1,0 & $0,987 \pm 0,005$ & $0,982 \pm 0,004$ \\
D & 1,0 & $0,967 \pm 0,004$ & $0,959 \pm 0,005$ \\
E & 1,0 & $0,976 \pm 0,004$ & $0,969 \pm 0,004$ \\
\hline F & 1,0 & $1,011 \pm 0,005$ & $1,000 \pm 0,005$ \\
\hline
\end{tabular}

\section{Conclusão}

O compósito SSF, produzido pelo processo sol-gel, é capaz de imobilizar e estabilizar a forma reduzida do azul de metileno de forma muito acentuada, pois é capaz de um deslocamento significativo do potencial formal do azul de metileno, quando comparados os valores da espécie eletroativa imobilizada e em solução.

A alta estabilidade eletroquímica do azul de metileno, quando imobilizado neste material microporoso com relação ao número de ciclos e ao efeito do $\mathrm{pH}$ do meio, sugere que este material apresenta em efeito protetor muito pronunciado. Pode-se supor que o azul de metileno esteja ancorado dentro de poros e estabilizado por múltiplas interações com os grupos ácidos dos óxidos metálicos ou grupos fosfato ácidos desta matriz.

Os estudos voltamétricos mostram que estes materiais não apresentam alta resistividade, ao contrário do que se espera, para um compósito com sílica em sua estrutura. Além disso, possibilita a 
eletrooxidação do ácido ascórbico no potencial de 0,0 $\mathrm{mV}$ vs ECS.

A utilização do eletrodo SSFAM como sensor eletroquímico para o ácido ascórbico em amostras reais demonstrou, em comparação com os resultados obtidos pelo método do 2,6-diclorofenilindofenol (método padrão), uma boa concordância entre os valores, sendo assim, o eletrodo quimicamente modificado SSFAM é altamente promissor como um sensor eletroquímico para vitamina $\mathrm{C}$ em amostras reais.

\section{Agradecimentos}

À Fundação Araucária pelo apoio financeiro e ao Prof. Yoshitaka Gushikem, do Instituto de Química da Unicamp, pelas análises de área superficial específica, volume médio dos poros e diâmetro médio dos poros.

\section{Referências}

ALFAYA, A. A. S., KUBOTA, L. T. A utilização de materiais obtidos pelo processo de sol-gel na construção de biossensores. Química Nova, São Paulo, v.25, n.5, p.835-841, 2002.

ANDREOTTI, E. I. S., GUSHIKEM, Y. Zirconium (IV) oxide coated silica gel surface as a base for copper hexacyanoferrate immobilization. Journal of the Brazilian Chemical Society, São Paulo, v.6, n.3, p.271-276, 1992.

CARDOSO, W. S.; FRANCISO, M. S. P.; LUCHO, A. M. S.; GUSHIKEM, Y. Synthesis and acidic properties of the $\mathrm{SiO}_{2} / \mathrm{SnO}_{2}$ mixed oxides obtained by the sol-gel process. Evaluation of immobilized copper hexacyanoferrate as an electrochemical probe. Solid State Ionics, Amsterdam, v.167, n.1/2, p.165-173, feb. 2004.

DAUFARTAS, M. F.; EVANS, J. F. E. C. Catalysis of ascorbic acid oxidation using plasma polymerized vinylferrocene film electrodes. Journal of
Electroanalytical Chemistry, Amsterdam, v.109, p.301-308, 1980.

FERREIRA, C. U.; GUSHIKEM, Y.; KUBOTA, L. T. Electrochemical properties of Meldola's Blue immobilized on sílica-titania phosphate prepared by the sol-gel method. Journal of New Materials for Electrochemical Systems, Montreal, v.6, n.4, p.298$303,2000$.

FUJIWARA, S. T.; GUSHIKEM, Y.; ALFAYA, R. V. S. Adsorption of $\mathrm{FeCl}_{3}, \mathrm{CuCl}_{2}$ and $\mathrm{ZnCl}_{2}$ on silsesquioxane 3-n-propylpyridiniumchloride polymer film adsorbed on $\mathrm{Al}_{2} \mathrm{O}_{3}$ coated silica gel. Colloids and Surfaces A, Amsterdam, v.178, n.1/3, p.135-141, mar. 2001.

GUSHIKEM, Y., ROSATTO, S. S. Metal oxide thin films grafted on silica gel surfaces: Recent advances on the analytical application of these materials. Journal of the Brazilian Chemical Society, São Paulo, v.12, n.6, p.695-700, nov./dec. 2001.

HARTLEY, F. Supported metal complexes. London: Dreider Publishing Company, 1985.

HORVATH, G.; KAWAZOE, K. Method for the calculation effective pore-size distribution in molecular-sieve carbon. Journal of Chemical Engineering of Japan, Tokyo, v.16, n.6, p.470-475, 1983.

KUBOTA, L. T.; GUSHIKEM, Y.; PEREZ, J., TANAKA, A. A. Electrochemical properties of iron phthalocyanine immobilized on titanium (iv) oxide coated on silica gel surface. Langmuir, Washington, v.11, p.1009-1013, 1995.

KURIHARA, L. A.; FUJIWARA, S. T.; ALFAYA, R. V. S.; GUSHIKEM, Y.; ALFAYA, A. A. S, CASTRO, S. C. Copper(II) adsorbed on $\mathrm{SiO}_{2} / \mathrm{SnO}_{2}$ obtained by sol-gel processing method: application as electrochemical sensor for ascorbic acid. Journal Of Colloid And Interface Science, New York, v.274, n.2, p.579-586, 2004. 
LINDQUIST, J. Voltametric determination ascorbic acid by use of a carbon paste electrode. Analyst, London, v.100, p.339-348, 1975.

MELO, L. F. C.; COLLINS, C. H.; COLLINS, K. E.; JARDIM, I. C. S. F. Stability of high-performance liquid chromatography columns packed with poly(methyloctylsiloxane) sorbed and radiationimmobilized onto porous silica and zirconized silica. Journal of Chromatography A, Amsterdam, v.869, p.129-135, 2000.

MELO, L. F. C.; JARDIM, I. C. S. F. Development of $\mathrm{C} 8$ stationary phases immobilized by $\gamma$ radiation on zirconized silica for high-performance liquid chromatographic applications. Journal of Chromatography A, Amsterdam, v.423, p.845-852, 1999.

MORITA, T.; ASSUMPÇÃO, R. M. V. Manual de soluções, reagentes e solventes. São Paulo: Edgard Blücher, 1972.

MUNTEANU, F. D.; KUBOTA, L. T.; GORTON, L. Effect of $\mathrm{pH}$ on the catalytic electrooxidation of NADH using two-electrons mediators immobilized on zirconium phosphate. Journal of Electroanalytical Chemistry and Interfacial Electrochemistry, Amsterdam, v.509, n.1, p.2-10, 2001.

PALECEK, E. From polarography of DNA to microanalysis with nucleic acid modified electrodes. Electroanalysis, New York, v.8, n.1, p.7-11, 1996.

PEREIRA, A. C.; KUBOTA, L. T. Otimização da preparação de eletrodo de pasta de carbono contendo riboflavina imobilizada em suporte inorgânico. Química Nova, São Paulo, v.27, n.5, p.725-729, 2004.

PEREZ, E. F.; NETO, G. D.; KUBOTA, L. T. Bienzymatic amperometric biosensor for oxalate. Sensors And Actuators B , Lausanne, v.72, n.1, p.8085,2001
PESSOA, C. A.; GUSHIKEM, Y.; KUBOTA, L. T.; GORTON, L. Preliminary electrochemical study of phenothiazines and phenoxazines immobilized on zirconium phosphate. Journal of Electroanalytical Chemistry, Amsterdam, v.431, n.1, p.23-27, 1997.

ROCHA, R. P.; ROSSATTO, S. S.; BRUNS, R. E.; KUBOTA, L. T. Factorial design optimization of redox properties of methylene blue adsorbed on a modified silica gel surface. Journal of Electroanalytical Chemistry and Interfacial Electrochemistry, Amsterdam, v.433, n.1/2, p.73-76, 1997.

ROLISON, D. R. Zeolita-modified electrodes and electrode-modified zeolites. Chemical Reviews, Washington, v.90, p.867-873, 1990.

ROSATTO, S. S., KUBOTA, L. T., NETO, G. D. Biosensor for phenol based on the direct electron transfer blocking of peroxidase immobilizing on silicatitanium. Analytica Chimica Acta, Amsterdam, v. 390, n.1-3, p.65-72, may 1999.

SILVA, R. B.; COLLINS, C. H. Chromatographic evaluation of radiation-immobilized poly (methylooctylsiloxane) on titanium-grafted silica. Journal of Chromatography A, Amsterdam, v.845, n.12, p.417-422, jun. 1999.

SILVA, R. B.; COLLINS, K. E.; COLLINS, C. H. Effects in high-performance liquid chromatography of high $\mathrm{pH}$ in the mobile phase on poly(methyloctylsiloxane) immobilized by $\gamma$ radiation on titanium-grafted silica. Journal of Chromatography A, Amsterdam, v. 869, n.12, p. 137-141, feb. 2000.

SILVA, R. B.; GUSHIKEM, Y.; COLLINS, C. H. Synthesis, characterization and chromatography evaluation of titanium tetrabutoxide-modified sílica as a support for HPLC-RP separation in alkaline mobile phases. Journal of Separation Science, Weinheim, v.24, n.1, p.49-54, jan. 2001. 
STANDARD METHODS FOR THE EXAMINATION OF WATER AND WASTEWATER. American Public Health Association. 18.ed. New York: American Water Works Association and Water Environment Federation, 1992.

WALCARIUS, A. Analytical applications of silicamodified electrodes - A comprehensive review. Electroanalysis, New York, v.10, n.18, p.1217-1235, dec.1998.

WEBB, P. A.; ORR, C. Analytical methods in fine particle technology. Norcross: Micrometrics Instrument Corp., 1997.

XING, X.; TAN, T.; SHAO, M.; LIU, C. Electrochemical oxidation of $\mathrm{L}$-ascorbic acid at the graphite electrode and its monitoring by a thick-film graphite sensor assembly. Electroanalysis, New York, v.4, n.2, p.191-197, 1992. 